\title{
TASK C: PARTICLE PHYSICS AND COSMOLOGY
}

\author{
Progress Report
}

for Period January 1988-October 1990

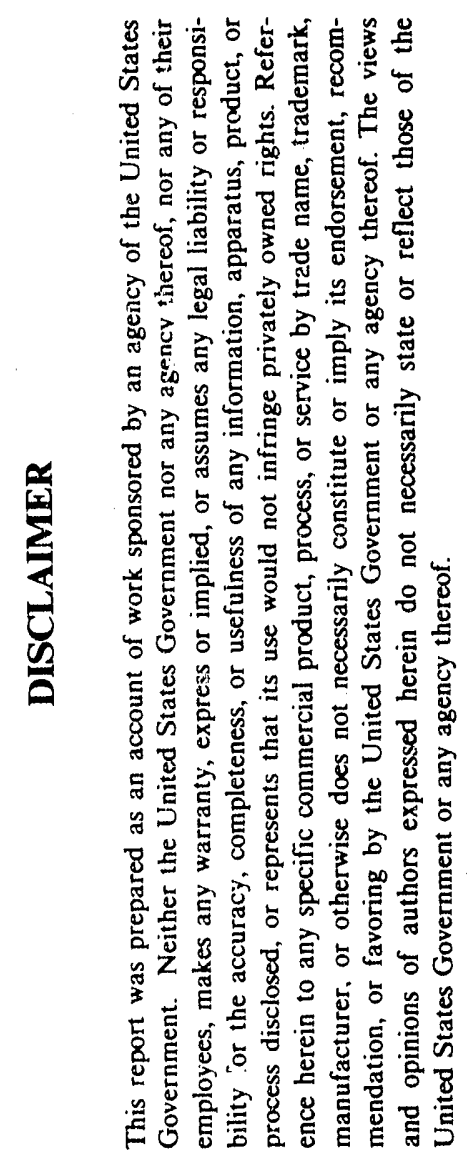

Michael S. Turner

The University of Chicago

5801 South Ellis Avenue

Chicago, Illinois 60637-1433

October 1990

Prepared for

THE U.S. DEPARTMENT OF ENERGY

GRANT NO. DE FG02-90ER-40560 


\section{RESEARCH ACCOMPLISHMENTS AND PUBLICATIONS}

\section{Inflationary Cosmology}

Inflation has revolutionized the way we think about the earliest history of the Universe. It provides a means for understanding the origin of the smoothness and flatness of the Universe, the origin of the seed inhomogeneities for structure formation, and a resolution to the monopole problem. ${ }^{1,2}$ In the past decade our group has made important contributions to the development of the inflationary paradigm: elucidating the mechanism for reheating in new inflation ${ }^{3}$ and for the origin of curvature ${ }^{4}$ and isocurvature ${ }^{5}$ perturbations. In addition, we have shown that inflation proceeds from very general initial conditions, ${ }^{6}$ we have proposed several models for inflation, ${ }^{7}$ including double inflation, ${ }^{8}$ and have addressed the production of gravitational waves, ${ }^{9}$ of large-scale primeval magnetic fields, ${ }^{10}$ and of massless particles. ${ }^{11}$

Last year La and Steinhardt proposed an extremely interesting new variant of inflation, called extended inflation..$^{12}$ Extended inflation allows inflation to be once again tied to a first-order, SSB phase transition. Extended inflation closely resembles Guth's original model of inflation, with one important difference: the bubble nucleation rate per Hubble volume per Hubble time $\left(\Gamma / H^{4}\right)$ increases with time rather than remaining constant $(\Gamma$ is the bubble nucleation rate and $H$ is the Hubble constant). This means that at early times, the nucleation rate can be small so that the Universe (or a small part of it) can become trapped in the false vacuum and inflates, and that at late times it can be large so that bubbles of true vacuum nucleate rapidly enough to complete the phase transition and reheat the Universe. In the original La-Steinhardt model, based upon the Jordan-BransDicke theory of gravity, the variation of $\Gamma / H^{4}$ came about because $H$ slowly decreased curing inflation (due to the evolution of the "gravitational constant"). While the original model is inconsistent with precision solar-system tests of gravity (sufficiently rapid bubble nucleation requires the Brans-Dicke parameter $\omega$ to be less than about $20,{ }^{13}$ while solar system tests require it to be greater than about 500 ), it provides an interesting toy model, the key new element being the variation of $\Gamma / H^{4}$.

We have analyzed the origin of density perturbations in extended inflation models, and showed that by a simple conformal transformation to the Einstein frame (where gravity is that of general relativity, but the couplings of iralar fields have been modified) extended inflation closely resembles new inflation and the formalism developed to analyze density perturbations in new inflation ${ }^{4}$ is directly apflicable. ${ }^{14}$ Moreover, the result is very encouraging, the amplitude of density perturbations is related to the ratio of the phase transition energy scale to the Planck scale to a power close to two, and for a phase transition energy scale of about $10^{14} \mathrm{GeV}$ density perturbations of the correct size arise. (In contrast, in new inflation, achieving density perturbations of an acceptable size required the inflaton field to be very, very weakly coupled, raising the issue of "fine tuning.") We also shnwed that isocurvature fluctuations can arise just as they do in new inflation. 
We have studied a number of other issues associated with extended inflation. Because the phase transition is completed by bubble nucleation, bubble collisions can have important effects. They lead to a new source of relic gravitational waves that are likely to be much more important (and more accessible to detection) than those produced by quantum fluctuations. ${ }^{15}$ Bubble collisions can also lead to the production of primordial black holes, ${ }^{16}$ whose evaporations can produce the baryon asymmetry of the Universe and are an additional new source of gravitational waves, and topological defects, such as monopoles and cosmic string. ${ }^{17}$ We have also studied the issue of baryogenesis, which can proceed very differently in extended inflation. ${ }^{18}$

The origin La-Steinhardt mode' points the way to a new and potentially very promising model of inflation, the key feature of which is the variation of the bubble nucleation rate per Hubble volume per Hubble time. It is also appealing because a generic prediction of superstring (and higher dimensional) theories is the existence of a Brans-Dicke-like field (or dilaton). However, as noted earlier, the La-Steinhardt model requires modification. We have considered the possibility that Brans-Dicke field becomes massive, ${ }^{14}$ which would remedy the conflict with solar system tests, or that extended inflation derives from a higher dimensional theory. ${ }^{19}$

\section{References}

1. A. Guth, Phys. Rev. D 23, 347 (1981).

2. A. D. Linde, Phys. Lett. B 108, 389 (1982); A. Albrecht and P. J. Steinhardt, Phys. Rev. Lett. 48, 1220 (1982).

3. A. Albrecht, P.J. Steinhardt, M.S. Turner, and F. Wilczek, Phys. Rev. Lett. 48, 1437 (1982); also see, L. Abbott, E. Fahri, and M. Wise, Phys. Lett. B 117 (1982); and A. Dolgov and A. Linde, Phys. Lett. B 116, 329 (1982).

4. J. M. Bardeen, P. J. Steinhardt, and M. S. Turner, Fhys. Rev. D 28, 679 (1983); also see, A. H. Guth and S.-Y. Pi, Phys. Rev. Lett. 49, 1110 (1982); A. A. Starobinskii, Phys. Lett. B 117, 175 (1982); and S. W. Hawking, Phys. Lett. B 115, 295 (1982).

6. J.A. Frieman and M.S. Turner, Phys. Rev. D 30, 265 (1984); M.S. Turner and L. Widrow, Phys. Rev. Lett. 57, 2237 (1986).

5. M. Axenides, R. Brandenberger, and M.S. Turner, Phys. Lett. B 128, 178 (1983); D. Seckel and M.S. Turner, Phys. Rev, D 32, 3178 (1985); A. D. Linde, Phys. Lett. $B$ 158, 375 (1985); M. S. Turner, A. Cohen, and D. Kaplan, Phys. Lett. B 216, 20 (1989).

6. M.S. Turner and L. Widrow, Phys. Rev. Lett. 57, 2237 (1986).

7. F. Accetta, D. Zoller, and M.S. Turner, Phys. Rev. D 31, 3046 (1985); P.J. Steinhardt and M.S. Turner, Phys. Rev. D 29, 2162 (1984).

8. J. Silk and M.S. Turner, Phys. Rev. D 35, 419 (1987).

9. M. T. Ressell and M. S. Turner, Phys. Rev. D, submitted (1990); also, see, V. A. Rubakov, M. Sazhin, and A. Veryaskin, Phys. Lett. B 115, 189 (1982); R. Fabbri 
and M. Pollock, Phys. Lett. B 125, 445 (1983); L. Abbott and M. Wise, Nucl. Phys. B244, 541 (1984); B. Allen, Phys. Rev. D 37, 2078 (1988).

10. M.S. Turner and L. Widrow, Phys. Rev. D 37, 2743 (1988).

11. M.S. Turner and L. Widrow, Phys. Rev. D 37, 3428 (1988).

12. D. La and P. J. Steinhardt, Phys. Rev. Lett. 62, 376 (1989); also see, D. La and P. J. Steinhardt, Phys. Lett. B 220, 375 (1989); D. La, P. J. Steinhardt, and E. Bertschinger, Phys. Lett. B 231, 231 (1989); F. S. Accetta and J. J. Trester, Phys. Rev. D 39, 2854 (1989); P. J. Steinhardt and F. S. Accetta, Phys. Rev. Lett. 64, 2740 (1990).

13. E. J. Weinberg, Phys. Rev. D 40, 3950 (1989).

14. E.W. Kolb, D. S. Salopek, and M. S. Turner, Phys. Rev. D, in press (1990).

15. M. S. Turner and F. Wilczek, Phys. Rev. Lett., submitted (1990).

16. J. D. Barrow, E. J. Copeland, E.W. Kolb, and A. R. Liddle, Phys. Rev. D, submitted (1990).

17. E. J. Copeland, E.W. Kolb, and A. R. Liddle, Phys. Rev. D, in press (1990).

18. J. D. Barrow, E. J. Copeland, E.W. Kolb, and A. R. Liddle, Phys. Rev. D, submitted (1990).

19. E.W. Kolb, R. Holman, S. L. Vadas, and Y. Wang), Phys. Rev. D, submitted (1990).

\section{Dark-matter Detection}

Most of the matter in the Universe is dark, i.e., does not give off any detectable radiation. ${ }^{1}$ Primordial nucleoysynthesis provides a stringent constraint to the fraction of critical density contributed by baryons: $\Omega_{B} \lesssim 0.1 .^{2}$ If $\Omega$ is greater than about 0.1 then the dark matter cannot be baryonic. Moreover, inflation "predicts" that the Universe is flat $(\Omega=1)$ and consideration of structure formation also strongly argues for $\Omega=1$. Thus, a line of sound reasoning leads one to consider seriously the possible existence of large amounts of nonbaryonic matter in the form of relic elementary particles. (The possibility that baryon number inhomogeneities produced by a strongly first-order quark/hadron transition would modify nucleosynthesis sufficiently to permit $\Omega_{B}=1.0,{ }^{3}$ no longer seems viabie. ${ }^{4}$ )

At present the three most promising dark matter candidates are the axion, the lightest supersymmetric partner (usually the neutralino), or a light neutrino species. (For purposes of structure formation the first candidates behave like cold dark matter, while the neutrino behaves like hot dark matter. ${ }^{5}$ At present, cold dark matter and inflation-produced curvature perturbations provides a very successful paradigm for structure formation.) We have been very active in studying the possibility that the dark matter in the Universe is either relic axions or neutralinos. We will discuss our work on axions in the following Section.

In order to calculate the relic abundance ${ }^{5}$ of neutralinos, the annihilation of neutralinos in the halo, earth, or sun, or the interactions of relic neutralinos with ordinary matter, one needs the annihilation cross section of two neutralinos into anything. For a light neu- 
tralino, mass less than $m_{W}$, the final states include fermion-antifermion pairs; for a heavy neutralino the final states include in addition $W W, Z Z, H W, H Z$. We have calculated the total annihilation cross section for the most general neutralino (a Higgsino/gaugino mixture)- light $^{6}$ or heavy ${ }^{7}$-in the minimal supersymmetric extension of the standard model. $^{8}$ Over most of parameter space $\left(\mu, M, \tan \beta, m_{\text {top }}\right.$, and the squark and slepton masses) relic neutralinos contribute at least $1 \%$ of critical density, and in many regions of parameter space they contribute critical density, making the neutralino a very attractive dark matter candidate. The lack of any evidence of supersymmetric in recent hadron and lepton collider experiments has generally pushed up the scale for supersymmetry and the mass of the neutralino. We have shown that the maximum cosmologically acceptable mass for a neutralino is $3.2 \mathrm{TeV}$ (a more massive neutralino would contribute too much mass density today; coincidentally, a neutralino heavier than this would not be attractive in the context of "low-energy" supersymmetry). ${ }^{7}$ (Along these same lines we have shown that there is a very general upper bound to the mass of a point-like particle relic based upon unitarity: to avoid "overclosing" the Universe, any stable, point-like species must be less massive than about $340 \mathrm{TeV} .{ }^{9}$ ) The cross sections that we have calculated are very important theoretical input for the direct and indirect detection of neutralino dark matter.

We have also explored two interesting and very distinctive signatures of dark matter annihilation in the galactic halo: mono-energetic positrons ${ }^{10}$ and mono-energetic photons. ${ }^{11}$ While the rates for both of these cosmic-ray signatures are likely to be too small to make them observable, they are worthy of note: If seen, they are a "smokin' gun" for particle dark matter in the halo.

\section{References}

1. J. Primack, et al., Ann. Rev. Nucl. Part. Sci. 38, 751 (1988); V. Trimble, Ann. Rev. Astron. Astrophys. 25, 425 (1987).

2. J. Yang, M.S. Turner, G. Steigman, D.N. Schramm, and K. Olive, Astrophys. J. 281, 493 (1984).

3. J. Applegate, C.J. Hogan, and R.J. Scherrer, Phys. Rev. D 35, 1151 (1987); also see, C. Alcock, G. Fuller, and G. Mathews, Astrophys. J. 320, 439 (1987); R.A. Malaney and W.A. Fowler, Astrophys. J. 333, 14 (1988).

4. See e.g., H. Kurkio-Sunio et al., Phys. Rev. D 38, 1091 (1988), or the review, H. Kurkio-Sunio et al., Astrophys. J. 353, 406 (1990).

5. See, e.g., E.W. Kolb and M.S. Turner, The Eanly Universe (Addison-W/esley, Redwood City, CA, 1990).

6. K. Griest, Phys. Rev. Lett. 61, 666 (1988); Phys. Rev. D 38, 2357 (1988); also see the earlier work, J. Ellis et al., Nucl. Phys. B 238, 453 (1984).

7. K. Griest, M. Kamionkowski, and M.S. Turner, Phys. Rev. D 41, 3565 (1990); also see, K. Olive and M. Srednicki, Phys. Lett. B 230, 78 (1989).

8. See, e.g., H. Haber and G. Kane, Phys. Rep. 117, 75 (1985). 
9. K. Griest and M. Kamionkowski, Phys. Rev. Lett. 64, 615 (1990).

10. M.S. Turner and F. Wilczek, Phys. Rev. D 42, 1001 (1990); also see, A.J. Tylka, Phys. Rev. Lett. 63, 840 (1989).

11. M. Srednicki, S. Thiessen, and J. Silk, Phys. Rev. Lett. 56, 263 (1986); S. Rudaz, Phys. Rev. Lett. 56, 2128 (1986); L. Bergstrom and H. Snellman, Phys. Rev. D37, 3737 (1988); L. Bergstrom, Nucl. Phys. B325, 647 (1989); G.F. Giudice and K. Griest, Phys. Rev. D 40, 2549 (1989); S. Rudaz, Phys. Rev. D 39, 3549 (1989); A. Bouquet, P. Salati, and J. Silk, Phys. Rev. D 40, 3168 (1989).

\section{Axions, Astrophysics, and Cosmology}

Peccei-Quinn (PQ) symmetry and its axicn is still the most attractive solution to the strong-CP problem, ${ }^{1}$ and therefore a most compelling and very minimal extension of the standard model. Axion models have one basic free parameter: the axion mass (or equivalently the $\mathrm{PQ}$-symmetry breaking scale, $m_{a} \simeq 0.6 \mathrm{eV} \cdot 10^{7} \mathrm{GeV} /\left(f_{P Q} / N\right)$ ), which a priori could have a value anywhere between about $10^{-11} \mathrm{eV}$ and $1 \mathrm{MeV}$. Laboratory experiments (in particular the unobserved decays $\mathrm{K}^{+} \rightarrow \pi^{+}+a, \Psi / J \rightarrow \gamma+a$, and $\Upsilon \rightarrow \gamma+a$ ) have ruled out an axion more massive than a few $\mathrm{keV}$. The rest-and bulkof the axion parameter space has been explored by use of the Heavenly Laboratory. At present two "windows" remain: $:^{2} 10^{-6} \mathrm{eV}$ to about $10^{-3} \mathrm{eV}$ and $3 \mathrm{eV}$ to $8 \mathrm{eV}$ (for the hadronic axion only).

The pioneering papers of $\mathrm{Sato}^{3}$ and of Kolb et al. ${ }^{4}$ pointed out that a light, weakly coupled particle like the axion could have a profound effect on the evolution of stars. Because axions interact so weakly, they provide an additional, very efficient means of transporting energy away from a star, and can thereby accelerate stellar evolution. (For a pedagogical discussion of the effect of axion emission on stars, see Frieman, Dimopoulos, and Turner. ${ }^{5}$ ) Very stringent limits to the axion mass based on the evolution of various types of stars-from the sun to red giants to white dwarfs to neutron stars-have been derived. ${ }^{2}$ Taken together, they preclude an axion more massive than about $10^{-3} \mathrm{eV}$, except for a small window around $3 \mathrm{eV}$ to $8 \mathrm{eV}$ (for hadronic axions only). This is an impressive improvement of almost seven orders of magnitude on laboratory experiment.

Two of the most stringent limits, that based upon the evolution of red giants and that based upon the cooling of SN 1987A, involved the work of our group. ${ }^{6,7}$ The constraint based upon SN 1987A is perhaps the most direct astrophysical limit, as the 19 anti-electron neutrino events provide a detailed record of the entire initial cooling phase of that newly born neutron star. The duration of the observed neutrino bursts-about 6 sec for IMB and about $12 \mathrm{sec}$ for KII-exclude an axion in the mass ranga of about $10^{-3} \mathrm{eV}$ to 3 $\mathrm{eV}$. (In particular, we have shown that the response of the KII and IMB detectors to the neutrino flux from a hot neutron star with axion cooling would lead to a burst duration of significantly less than this for an axion in this mass range. ${ }^{8}$ )

It may seem surprising that the SN 1987A data do not exclude an axion heavier than 
about $3 \mathrm{eV}$, as the coupling strength of an axion increases with its mass. The reason is simple and remarkable: the core of the newly born neutron star is opaque to axions more massive than about $10^{-2} \mathrm{eV}$, so that an axion heavier than this is radiated from an "axion sphere." An axion more massive than about $3 \mathrm{eV}$ interacts so strongly within the core that their effect on the cooling of the core is not significant. (In treating the so-called trapping regime, we computed the axion opacity of nuclear matter(!), and incorporated full axion transport into detailed models of the initial cooiling of the newly born nentron star. ${ }^{8}$ )

Axions arise as cosmological relics through three distinct mechanisms: (1) As thermal relics-axions more massive than about $10^{-3} \mathrm{eV}$ come into thermal equilibrium through pion-axion conversion, ${ }^{9} \pi+N \rightarrow a+N$ ( $N$ is a nucleon), and have a relic abundance comparable to that of a light neutrino species, about $30 \mathrm{~cm}^{-3}$. (2) The misalignment process-at high temperatures $\left(\gg \Lambda_{\mathrm{QCD}}\right.$ ) the free energy of the QCD vacuum becomes independent of the axion angle, and the value of the axion angle through the Universe will, in general, be misaligned with the minimum of the free energy at low temperatures. When the Universe cools to temperatures of order a $\mathrm{GeV}$, the free energy develops a minimum at the $C P$-conserving value of the axion angle due to instanton effects, and throughout space the axion field will be misaligned with this minimum, and hence will begin to oscillate. These oscillations correspond to a condensate of very low-momentum axions. ${ }^{10}$ (3) Axionic string decay - if the Universe did not inflate or inflated before PQ-symmetry breaking, then the breaking of $\mathrm{PQ}$ symmetry also leads to the production of axionic strings, which ultimately decay and produce large numbers of axions-comparable to or greater than the number produced by the misalignment mechanism (the precise number is still a matter of intense debate). ${ }^{11}$

Thermal axions cannot close the Universe. However, the decays of multi-eV thermal axions that reside in rich clusters offer a means of detecting such axions. We applied for telescope time at Kitt Peak to carry out such a search, received the telescope time, and carried out the observations last May. While the data analysis is not quite complete, we have ruled out an axion more massive than $5 \mathrm{eV}$; with the data in hand we will be able to search for an axion as light as about $3.1 \mathrm{eV} .{ }^{12}$ In the end we should be able to explore the entire multi-eV window.

The coherently produced axions can close the Universe, for an axion mass in the range of about $10^{-6} \mathrm{eV}$ to about $10^{-4} \mathrm{eV}$ (and perhaps as high as $10^{-3} \mathrm{eV}$ if axionic string decay is very prolific). Three first-generation "Sikivie detectors" have been built to search for cosmic axions in our halo in this mass range. ${ }^{13}$ While these first generation detectors did not have sufficient sensitivity to detect halo axions (if the axion has the canonical coupling to two photons), they did establish the feasibility of the concept. A second generation detector: with the required sensitivity to explore the mass range from about $10^{-6} \mathrm{eV}$ to $10^{-4} \mathrm{eV}$ has been proposed, ${ }^{14}$ and we are part of this collaboration.

We have addressed a number of issues regarding the relic abundance of cosmic axions in the $10^{-6} \mathrm{eV}$ to $10^{-4} \mathrm{eV}$ mass range. We carried out the most detailed calculation of 
the relic aburidance due to the misalignment mechanism, ${ }^{15}$ studied various theoretical uncertainties including entropy production and the effects of the QCD phase transition, ${ }^{16}$ and recently discussed the additional cosmological constraints that apply if the Universe inflated after PQ-symmetry breaking. We have also studied the periodic signatures-due to the earth's orbital and rotational motions--that arise in the detection of relic axions and that can be used to confirm the existence of axions in the halo and to probe the distribution of material in the halo. ${ }^{17}$

\section{References}

1. For a review of the axion, see, e.g., R.D. Peccei, in CP Violation, ed. C. Jarlskog (WSPC, Singapore, 1989).

2. For a review of the astrophysical and cosmological constraints, see, e.g., M.S. Turner, Phys. Rep., in press (1990); or G.G. Raffelt, Phys. Rep., in press (1991).

3. K. Sato, Prog. Theor. Phys. 60, 1942 (1978).

4. D.A. Dicus, et al., Phys. Rev. D 18, 1829 (1978).

5. J.A. Frieman, S. Dimopoulos, and M.S. Turner, Phys. Rev. D 36, 2211 (1987).

6. D.S.P. Dearborn, D.N. Schramm, and G. Steigman, Phys. Rev. Lett. 56, 26 (1986); G.G. Raffelt and D.S.P. Dearborn, Phys. Rev. D bf 36, 2201 (1987).

7. M.S. Turner, Phys. Rev. Lett. 60, 1797 (1988); A. Burrows, M.S. Turner a.d R.P. Brinkmarn, Phys. Rev. D 39, 1020 (1989); R.P. Brinkmann and M.S. Turner, Phys. Rev. D 38, 2338 (1988); M.S. Turner, H.-S. Kang, and G. Steigman, Phys. Rev. D 40, 299 (1989); A. Burrows, M.T. Ressell, and M.S. Turner, Phys. Rev. D, in press (1990); R. Mayle, et al., Phys. Lett. B 203, 188 (1988); ibid 219B, 515 (1989); G.G. Raffelt and D. Seckel, Phys. Rev. Lett. 60, 1793 (1988); K. Choi, K. Kang, and J.-E. Kim, Phys. Rev. Lett. 62, 849 (1989); N. Iwamoto, Phys. Rev. D 39, 2120 (1989); M. Carena and R.D. Peccei, Phys. Rev. D 40, 652 (1989); T. Hatsuda and M. Yoshimura, Phys. Lett. B 203, 469 (1988); T.E.O. Ericson and J.-F. Mathiot, Phys. Lett. B 219, 515 (1989); J. Engel et al., Phys. Rev. Lett. 65, 960 (1990).

8. A. Burrows, M.T. Ressell, and M.S. Turner, Phys. Rev. D, in press (1990). The freely streaming regime $\left(m_{a} \lesssim 10^{-2} \mathrm{eV}\right.$ is treated in A. Burrows, R.P. Brinkmann, and M.S. Turner, Phys. Rev. D. 39, 1020 (1989).

9. M.S. Turner, Phys. Rev. Lett. 59, 2489 (1987).

10. J. Preskill, M. Wise, and F. Wilczek, Phys. Lett. B 120, 127 (1983); L. Abbott and P. Sikivie, Phys. Lett. B 120, 133 (1983); M. Dine and W. Fischler, Phys. Lett. B 120, 137 (1983).

11. R. Davis, Phys. Lett. B 180, 225 (1986); D. Harrari and P. Sikivie, Phys. Lett. B 195, 361 (1987); R. Davis and E.P.S. Shellard, Nucl. Phys. B324, 167 (1990); A. Dabholkar and J.M. Quashncik, Nucl. Phys. B 333, 815 (1990); C. Hagmann and P. Sikivie, Nucl. Phys., in press (1990).

12. M. Bershady, M.T. Ressell, and M.S. Turner, Phys. Rev. Lett., submitted (1990). 
13. S. DePanfilis et al., Phys. Rev. Lett. 59, 839 (1987); Phys. Rev. D 40, 3153 (1989); C. Hagmann, P. Sikivie, N.S. Sullivan, and D.B. Tanner, Phys. Rev. $D(R C) \mathbf{4 2}, 1297$ (1990); Rev. Sci. Instrum. 61, 1076 (1990); S. Inagaki et al., in Proceedings of the Workshop on Cosmic Axions, edited by C. Jones and A. Melissinos (WSPC, Singapore, 1990).

14. P. Sikivie et al., Proposal to the DOE and LLNL for an Experimental Search for Dark Matter Axions in the 0.6-16 $\mu \mathrm{eV}$ Mass Range (submitted 20 July 1990).

15. M.S. Turner, Phys. Rev. D 33, 889 (1986).

16. M.S. Turner, Phys. Rev. D 32, 843 (1985); P.J. Steinhardt and M.S. Turner, Phys. Lett. B 129, 51 (1983); also see, W. Unruh and R. Wald, Phys. Rev. D 32, 831 (1985); T. Degrand, T.W. Kephart, and T.J. Weiler, Phys. Rev. D 33, 910 (1986); G. Lazarides, D. Panagiotakapoulos, and Q. Shafi, Phys. Lett. B 192, 323 (1987); K. Yamamoto, Phys. Lett. B 161, 289 (1985); S. Dimopoulos and L. Hall, Phys. Rev. Lett. 60, 1899 (1988).

17. M.S. Turner, Phys. Rev. D 42, in press (1990).

\section{Topological Defects}

Perhaps the most interesting consequence of spontaneously broken gauge theories is the fact that at high temperatiocs broken symmetries are stored. $\dagger$ Because of the high temperatures that existed during the earliest history of the Universe, there should be several-if not many-symmetry-breaking phase transitions, e.g., chiral symmetry breaking, electroweak symmetry breaking, and GUT symmetry breaking (?). The most interesting cosmological consequence of such phase transitions is the possibility that these transitions proceed "imperfectly" and that topological (and nontopological) defects are left behind as relics. There are a variety of topological defects possible: ${ }^{1}$ Suppose that the group $\mathcal{G}$ is broken down to the smaller group $\mathcal{H}$; magnetic monopole solutions exist if $\Pi_{2}(\mathcal{M}) \neq \mathcal{I}_{;}$(cosmic) string solutions exist if $\Pi_{1}(\mathcal{M}) \neq \mathcal{I}_{\text {; }}$ and domain wall solutions exist if $\Pi_{0}(\mathcal{M}) \neq \mathcal{I}$ (where $\mathcal{M}=\mathcal{G} / \mathcal{H}$ is the manifold of vacuum states). (If $\mathcal{G}$ is a global symmetry, then textures are also possible, provided that $\Pi_{3}(\mathcal{M}) \neq \mathcal{I}_{\text {; see, e.g., Ref. 2.) }}$ The existence of particle horizons in the standard cosmology preclude correlations on scales larger than about $c t$, and so if a topological soliton solution exists, of order one per horizon volume should be produced during the symmetry-breaking phase transition, through the so-called Kibble mechanism. ${ }^{3}$

Through the years our group has been very active in exploring the astrophysical and cosmological consequences of topological defects produced in the early Universe. We derived very stringent constraints to the flux of relic magnetic monopoles based upon the survival of astrophysical magnetic fields, ${ }^{4}$ and monopole catalysis of nucleon decay in various astrophysical objects. ${ }^{5}$ By the use of variational solutions, we have studied the in

$\dagger$ Of course, there are examples of the converse, where the full symmetry is restored at low temperatures. 
detail the properties of cosmic strings, both ordinary and superconducting. ${ }^{6}$ We have also studied the important issue of whether or not superconducting cosmic string solutions arise in realistic models, ${ }^{7}$ and the phase transition that occurs when the critical current is exceeded. ${ }^{8}$ We have explored the possibility that cosmic string loops could seed structure formation in the Universe, including the first numerical simulation structure formation seeded by cosmic string loops. ${ }^{9}$

Two of the most recent Ph.D. theses produced by students in our group have involved numerical simulations of phase transitions. Hodges carried out real-time simulations of scalar field theories that support domain wall and cosmic string solutions by numerically solving the classical field equations with an eppropriate noise term to simulate the effects of the thermal bath. His work was the first real-time simulation of a phase transition. While none of the results were unexpected, Hodges' work put the standard lore for the production of topological defects (the Kibble mechanism) on a very firm basis. ${ }^{10}$

Kawano's thesis lealt with the formation and early evolution of a domain wall network. Rather then solving the classical field equations, he used the thin-wall approximation, deriving the equations of motion for the wall from a generalized Nambu action. His work indicates that shortly after the domain wall network forms, it consists of a single wall that extends across the horizon, with very few vacuum bubbles (closed regions of one vacuum state surrounded by the other vacuum state). Moreover, he found that because of the finite velocity of the walls the energy density in walls evolves as $R^{-4 / 3}$ rather than $R^{-1}$, which would pertain if the walls were motionless $(R=$ the cosmic scale factor $) .{ }^{11}$

Many classical field theories admit non-topological soliton solutions. They were first discussed a number of years ago by Rosen, and by Friedberg, Lee, and Sirlin. ${ }^{11}$ They have more recently been studied (and called Q-balls) by Coleman. ${ }^{13}$ Unlike topological solitons such as magnetic monopoles, cosmic strings, and domain walls that arise in spontaneously broken gauge theories with nontrivial vacuum topology, non-topological solitons (NTS's) exist as the result of a conserved Noether ch.arge carrier by some field cor.fined to a finite region of space (and can even arise in the electroweak model ${ }^{14}$ ). NTS's have also recently been considered in theories with cosmic neutriıo balls, ${ }^{15}$ quark nuggets, ${ }^{16}$ and soliton stars. ${ }^{17}$ We have studied many aspects of NTS's, including their formation and subsequent evolution. ${ }^{18}$

\section{References}

1. See, e.g., A. Vilenkin, Phys. Rep. 121C, 264 (1984).

2. See, e.g., N. Turok, Phys. Rev. Iett. E3, 2625 (1989), and references therein.

3. T.W.B. Kibble, J. Phys. A 9, 1387 (1976).

4. M.S. Turner, E.N. Parker, and T. Bogdan, Phys. Kiev. D 26, 1296 (1982); Y. Raphaeli and M.S. Turner, Phys. Lett. B 121, 115 (1983).

5. E.W. Kolb, S. Colgate, and J.A. Harvey, Phys. Rev. Lett. 49, 1373 (1982); K. Freese, M.S. Turner, and D.N. Schrsmm, Phys. Rev. Lett. 51, 1625 (1983); S. Dimopoulos, 
J. Preskill, and F. Wilczek, Phys. Lett. B 119, 320 (1982); K. Freese, Astrophys. J. 286, 216 (1984); E.W. Kolb and M.S. Turner, Astrophys. J. 286, 702 (1984).

6. C.T. Hill, H.M. Hodges, and M.S. Turner, Phys. Rev. Lett. 59, 2493 (1987); Phys. Rev. D 37, 263 (1988).

7. L.M. Widrow, Phys. Rev. D 38, 1684 (1988).

8. F. Accetta, Nucl. Phys. B 313, 660 (1989).

9. R. Brandenberger, N. Kaiser, N. Turok, and D.N. Schramm, Phys. Rev. Lett. 59, 2371 (1987); R.J. Scherrer, Astrophys. J. 320, 1 (1987); A. Melott and R.J. Scherrer, Nature 328, 691 (1987); R.J. Scherrer et al., Phys. Rev. Lett. 62, 379 (1989).

10. H.M. Hodges, Phys. Rev. D 39, 3557 (1989); also see more recent work by W. Press, B.S. Ryden, and D.N. Spergel, Astrophys. J. 347, 590 (1989).

11. L. Kawano, Phys. Rev. D 41, 1013 (1990).

12. G. Rosen, J. Math. Phys. 9, 996 (1968); R. Friedberg, T. D. Lee, and A. Sirlin, Phys. Rev. D 13, 2739 (1976).

13. S. Coleman, Nucl. Phys. B 262, 263 (1935).

14. E. Copeland, K. Lee, and E. W. Kolb, Nucl. Phys. B 319, 501 (1989).

15. B. Holdom, Phys. Rev. D 36, 1000 (1987).

16. E. Witten, Phys. Rev. D 30, 272 (1984).

17. T. D. Lee, Phys. Rev. D 35, 3637 (1987).

18. J. A. Frieman, G. Gelmini, M. Gleiser, and E. W. Kolb, Phys. Rev. Lett. 60, 2101 (1988); E. Copeland, E. W. Kolb, and K. Lee, Phys. Rev. D 38, 3023 (1988); G. Gelmini, M. Gleiser, and E. W. Kolb, Phys. Rev. D 39, 1558 (1989); K. Griest and E. W. Kolb, Phys. Rev. D 40, 3231 (1989); K. Griest, E. W. Kolb, and A. Massarotti, Phys. Rev. D 40, 3529 (1989).

\section{Astrophysical/Cosmological Constraints to Particle Properties}

Over the past decade members of our group have used various apparatus in the Heavenly Laboratory-including SN 1987A, primordial nucleosynthesis, the galactic magnetic field, the sun, red giants, and neutron stars-to explore regic:- of particle-property parameter space that are beyond the reach of terrestrial laboratories to place very stringent limits to the properties of neutrinos (mass, lifetime, magnetic moment, chemical potential, number of flavors, and secret interactions), superheavy magnetic monopoles (relic abundance), neutralinos, and axions. ${ }^{1}$ The most well known of these limits, the limit to the number of flavors of light (mass less than about $1 \mathrm{MeV}$ ) neutrino flavors: $N_{\nu} \leq 4,{ }^{2}$ has recently been confirmed by experiments at both SLC and LEP; the current determination of the number of neutrino flavors, based upon $Z^{0}$ boson production and decay is $N_{\dot{\nu}}=3.0 \pm 0.1{ }^{3}$ which is a striking confirmation of this cosmological constraint and further verification of our excellent understanding of the Universe at $1 \mathrm{sec}$ after the bang. 


\section{References}

1. For a summary, see e.g., E.W. Kolb and M.S. Turner, The Early Universe (AddisonWesley, Redwood City, CA, 1990), Chs. 4, 7, 10; M.S. Turner, Ph.ys. Rep., in press (1990); and E.W. Kolb, D.N. Schramm, and M.S. Turner, in Neutrino Physics, ed. K. Winter (Cambridge Univ. Press, Cambridge, 1991).

2. G. Steigman, D.N. Schramm, and J. Gunn, Phys. Lett. 66B, 202 (1977); J. Yang et al., Astrophys. J. 281, 493 (1984); G. Steigman, K.A. Olive, D. Schramm, and M.S. Turner, Phys. Lett. 176B, 33 (1986).

3. As reported by the ALEPH, DELPHI, L3, OPAL Collaborations at the 25th International Meeting on High-energy Physics (Singapore, August 1990).

\section{Other Activities}

In addition to the research activities discussed above, members of our group have been involved in many other smaller projects. For example, we analyzed and discussed the nonadiabatic MSW solution to the solar neutrino problem. ${ }^{1}$ Taken at face value, the SAGE nonobservation of low-energy solar neutrinos ${ }^{2}$ favors this solution with its distinction signature of very few low-energy solar neutrinos. Since the SAGE results are consistent with the standard solar model at the $2 \sigma$ level, it is prudent to wait for more definitive results, which should be forthcoming soon from both the SAGE and GALLEX. experiments.

Motivated by the theoretical speculation of Hill and Ross ${ }^{3}$ that there could be other, even lighter pseudo-Nambu-Goldstone hosons in addition to the axion, we have considered the possible cosmological effects, including a late-time phase transition that could seed the formation of large-scale structure, ${ }^{4}$ or temporal oscillations in some of the fundamental constants, ${ }^{5}$ which would lead to apparent spatial periodicity in the Universe (similar to that recently reported by Broadhurst et al. ${ }^{6}$ ). Needless to say, both of these possibilities are highly speculative!

There is a growing consensus that nonperturbative effects associated with field configurations known as sphalerons lead to rapid violation of fermion number $(B+L)$ in the standard electroweak theory at temperatures around $200 \mathrm{GeV}$ to $300 \mathrm{GeV}$, and probably at all temperatures well above the phase transition. ${ }^{7}$ This has important implications for the origin of the baryon asymmetry of the Universe: Either it must be produced at temperatures below about $200 \mathrm{GeV}$ or the baryon asymmetry produced must have associated with it a nonzero value of $B-L$. We have taken the first steps towards a detailed re-examination of baryogenesis, and have shown that if the latter is true, then there are severe constraints to neutrino masses (if they are generated by the see-saw mechanism). ${ }^{8}$

\section{References}

1. E.W. Kolb, M.S. Turner, and T.P. Walker, Phys. Lett. B 175, 438 (1986); also see, L. Wolfenstein, Phys. Rev. D 17, 2369 (1979); S.P. Mikheyev and A.Yu. Smirnov, Sov. J. Nucl. Phys. 42, 918 (1986); H.A. Bethe, Phys. Rev. Lett. 56, 1305 (1986); S.P. Rosen and J.M. Gelb, Phys. Rev. D 34, 969 (1986). 
2. As reported by the SAGE (Soviet American Gallium Exper rent) collaboration at Neutrino ' 90 and The 25th International Conference on High-energy Physics (summer 1990).

3. C. Hill and G. G. Ross, Phys. Lett. 203B, 125 (1988); Nucl. Phys. B311, 253 (1988).

4. C. Hill, J. N. Fry, and D. N. Schramm, Comments on Nucl. Part. Phys. 19, 25 (1989); A. Stebbins and M.S. Turner, Astrophys. J. 339, L13 (1989); A. Massarotti, Phys. Rev. D, in press (1990); J.A. Frieman, C. Fill, and R. Watkins, Phys. Rev. D, submitted (1990); M.S. Turner, R. Watkins, and L.M. Widrow, Astrophys. J., submitted (1990).

5. M.S. Turner and C.T. Hill, FERMILAB-PUB-90/103-T (1990); C.T. Hill, P.J. Steinhardt, and M.S. Turner, Phys. Lett. B, in press (1990); also see, M. Morikawa, Univ. Brit. Columbia preprints 90-0208 and 90-0380 (1990).

6. T.J. Broadhurst, R. Ellis, D. Koo, and A.S. Szalay, Nature 343, 726 (1990).

7. V.A. Kuzmin V.A. Rubakov, and M.E. Shaposhnikov, Phys. Lett. B 155, 36 (1985); for a recent review of the subject see, V.A. Matveev, V.A. Rubakov, A.N. Tavkinelidze, and M.E. Shaposhnikov, Usp. Fiz. Nauk. 156, 253 (1988); M.E. Shaposhnikov, in Proc. XXIV Intl. Conf. on High-energy Physics (Munich, 1988); or Proc. on the Workshop on Sphalerons and Related Topics, ed. L. McLerran (Univ. of Minn., 1988); D.Yu. Grigoriev, V.A. Rubakov, and M.E. Shaposhnikov, Phys. Lett. B 216, 172 (1989); J. Ambjorn, T. Askgaard, H. Porter, and M.E. Shaposhnikov, Phys. Lett. B 244, 479 (1990).

8. J.A. Harvey and M.S. Turner, Phys. Rev. D, in press (1990).

\section{PUBLICATIONS SUPPORTED BY THIS GRANT (since January 1988)}

1. Primordial Nucleosynthesis with Decaying Particles. I. Entropy-producing Decays Robert J. Scherrer and Michael S. Turner Astrophysical Journal 331, 19 (1988)

2. Primordial Nucleosynthesis with Decaying Particles. II. Inert Decays Robert J. Scherrer and Michael S. Turner Astrophysical Journal 331, 33 (1938).

3. Inflation in the Universe Michael S. Turner Acta Physica Hungarica 64, 285 (1988).

4. Toward the Inflationary Paradigm: 3 Lectures on Inflation Michael S. Turner in Proceedings of the Erice School on Gauge Theory and the Early Universe, eds. P. Galeotti and D.N. Schramm (Kluwer, Dordrecht, 1989).

5. Gravitational Production of Scalar Particles in Inflationary Universe Models Michael S. Turner and L. Widrow 
Physical Review D37, 3428 (1988).

6. Inflation-produced, Large-scale Primeval Magnetic Fields

Michael S. Turner and L. Widrow

Physical Review D37, 2743 (1988).

7. Superconducting Cosmic Strings and the Photofission of ${ }^{4} \mathrm{He}$ in the Early Universe

H.M. Hodges, J. Silk, and Michael S. Turner

FERMILAB-PUB-87/153-A (1987).

8. On the Magnitude of Baryon-to-Photon Ratio Inhomogeneities Resulting from a First Order Quark/Hadron Transition

Michael S. Turner

Physical Review D37, 3049 (1988).

9. Axions and SN $1987 \mathrm{~A}$

Michael S. Turner

Physical Review Letters 60, 1797 (1988).

10. Primordial Lithium: New Reaction Rates, New Abundances, and New Constraints

L. Kawano, D. Schramm, and G. Steigman

Astrophysical L':rnal 327, 750 (1988).

11. Consistency between Dark Matter, Large Structure, and Galaxy Formation.

D.N. Schramm

High-Energy Physics in the 1990's: Snowmass 1988, ed. S. Jensen (WSPC, Singapore, 1989).

12. Constraints on Axions from SN 1987A

R. Mayle, J.Wilson, J. Ellis, K. Olive, D. Schramm, and G. Steigman

Physics Letters 203B, 188 (1988).

13. Cosmological QCD, Neutron Diffusion, and the Production of Primordial Heavy Elements

J. Applegate, C. J. Hogar and R. J. Scherrer

Astrophys. J. 329, 572 (1988).

14. Are Cosmic Strings Frustrated?

C. Hill, A. Kagan, and L. M. Widrow

Physical Review D 38, 1100 (1988).

15. Domain Walls with Strongly-bound Bose Condensates

H. M. Hodges

Physical Review D 37, 3052 (1988).

16. Effects of the Sun's Gravity on the Distribution of Dark Matter Near the Earth

K. Griest

Physical Review D 37, 2703 (1988).

17. Particle Cosmology Comes of Age

M. S. Turner 
Proc. of the 1987 International Symposium on Lepton and Photon Interactions at High Energies, eds. W. Bartel and R. Rückl (North-Holland, Amsterdam, 1988;.

18. Limits to the Radiative Decays of Neutrinos and Axions from $\gamma$-Ray Observations of SN 1987A

E. W. Kolb and M. S. Turner

Physical Review Letters 62, 509 (1989).

19. Phase Transitions in Superconducting Cosmic Strings

F.S. Accetta

Nuclear Physics B 313, 660 (1989).

20. Ordinary and Superconduction Cosmic Strings: A Variational Analysis

H. M. Hodges

in Proceedings of the Yale Cosmic String Woribshop, eds. F. Accetta and L.M. Krauss (World Scientific, Singapore, 1989).

21. String and Wall Formation in the Early Universe

H. M. Hodges

Physics A 158, 479 (1989).

22. Monte Carlo Model of Domain-wall Formation

H. M. Hodges

Physical Review D 39, 3557,1989$)$.

23. The Coasting Cosmology

E.W. Kolb

Astrophysical Journal 344, 543 (1989).

24. Zero Modes and Anomalies in Superconducting Strings

L. M. Widrow

Physical Review D 38, 1684 (1988).

25. Gauged Q-Balls

K. Lee, J. A. Stein-Schabes, R. Watkins, and L. M. Widrow

Physical Review D 39, 1665 (1988).

26. Genere' Relativistic Domain Walls

L. M. Widrow

Physical Review D 39, 3571 (1989).

27. Effect of the Sun's Gravity on the Distribution and Detection of Dark Matter Near the

Earth

K. Griest

Physical Review D 37, 2703 (1988).

28. Calculations of Rales for Direct Detection of Neutralino Dark Matter

K. Griest

Physical Review Letters 61, 666 (1988).

29. Cross Sections, Relic Abundance, and Detection Rates for Neutralino Dark Matter K. Griest 
Physical Review D 38, 2357 (1988).

30. Neutralino Dark Matter and its Detection

K. Griest

Proceedings of the Erice School on Dark Matter, eds. P. Galeotti and D. N. Schramm (Kluwer, Dordrecht, 1989).

31. Lower Bound on $e^{+} e^{-}$Decay of Massive Neutrinos

R. Cowsik, D. Schramm, and P. A. Hoflich

Physics Letters B 218, 91 (1989).

32. Cosmological Structure Formation from Soft Topological Defects

C. Hili, D. $\therefore$. Schramm, and J. N. Fty

Comments on Nuclear and Particie Physics 19, 25 (1988).

33. Primordial Origin of Nontopological Solitons

J. A. Frieman, G. Gelmini, M. Gleiser, and E. W. Kolb

Physical Review Letters 60, 2101 (1988).

34. Nontopological Cosmic Strings

E. Copeland, E. W. Kolb, and K. Lee

Physical Review D 38, 3023 (1988).

35. Cosmology of Biased Discrete Symmetry Breaking

G. Gelmini, M. Gleiser, and E. W. Kolb

Physical Review D 39, 1558 (1989).

36. Ground State of High-density Matter

E. Copeland, K. Lee, and E. W. Kolb

Nuclear Physics B 319, 501 (1989).

37. Solitosynthesis: Cosmological Evolution of Nontopological Solitons

K. Griest and E. W. Kolb

Physical Review D 40, 3231 (1989).

38. Guide to Primordial Nucleosynthesis Programming

L. Kawano

FERMILAB-PUB/34-A (March 1988).

39. Isocurvature Baryon Number Fluctuations in and Inflationary Universe

M. S. Turner, A. Cohen, and D. Kaplan

Physics Letters B 216, 20 (1989).

40. Numerical Rates for Nucleon, Nucleon Axion Bremsstrahlung

R. P. Brinkmann and M. S. Turner

Physical Review D 38, 2338 (1988).

41. Axions and SN 1987A

A. Burrows, M. S. Turner, and R. P. Brinkmann

Pirysical Review D 39, 1020 (1989).

42. History of Elementary Particle Physics

T. Appelquist, M. Gell-Mann, F. Gilman, P. Ramond, and M. S. Turner 
International Journal of Modern Physics A, in press (1990).

43. Axions and SN 1987A

M. S. Turner

Proceedings of the Storrs Meeting, eds. K. Haller, et al. (WSPC, Singapore, 1989).

44. Axions, SN 1987A, and One-Pion Exchange

M. S. Turner, H.-S. Kang, and G. Steigman

Physical Review D 40, 299 (1989).

45. The Grand Unified Photon Spectrum: A Coherent View of the Diffuse Extra-galactic

Radiation

M. T. Ressell and M. S. Turner

Comments on Astrophysics XIV, 323 (1990).

46. Relic Gravitational Radiation from Inflation

M. T. Ressell and M. S. Turner

Physical Review D, submitted (1990).

47. Particle Cold Dark Matter

E. W. Kolb and M. S. Turner

High-energy Physics in the 1990's: Snowmass 1988, ed. S. Jensen (WSPC, Singapore, 1989).

48. Is the Great Attractor Really a Great Wall?

A. Stebbins and M. S. Turner

Astrophys. J. (Letters) 339, L13 (1989).

49. Model Independence of Constraints on Particle Cold Dark Matter Candidates

K. Griest and B. Sadoulet

in Erice School on Dark Matter in the Universe, eds. P. Galeotti and D. N. Schramm (Kluwer, Dordrecht, 1989).

50. The Effects of Cosmions Upon the Structure and Evolution of Very Low Mass Stars

E. E. Deluca, K. Griest, R. Rosner and J. Wang

FERMILAB-Pub-89/49-A (1989).

51. Statistical Fluctuations as the Origin of Nontopological Solitons

K. Griest, E. W. Kolb, and A. Massarotti

Physical Review D 40, 3529 (1989).

52. Rate for Annihilation of Galactic Dark Matter into Two Photons

K. Griest and G. F. Guidice

Physical Review D 40, 2549 (1989).

53. Phenomenology and Cosmology of Extra Generations of Higgs Bosons

K. Griest and M. Sher

Physical Review Letters 64, 135 (1990).

54. Monopoles and Main Sequence Stars

J. A. Frieman, K. Freese and Michael S. Turner

Ap.J. 335, 844 (1988). 
55. Positron-Line Radiation from Halo WIMP Annihilations as a Dark Matter Signature Michael S. Turner and F. Wilczek

Physical Review D 42, 1001 (1990).

56. Eternal Annihilations

J. A. Frieman, E. W. Kolb, and Michael S. Turner

Physical Review D 41, 3080 (1990).

57. Windows on the Axion

Michael S. Turner

Physics Reports, in press (1990).

58. A New Probe of the Homogeneity of the Universe

J.A. Frieman and Michael S. Turner

FERMILAB-PUB-89/267-A (1989).

59. Windows on the Axion

Michael S. Turner

in Proceedings of the Workshop on Cosmic Axions, ed. C.Jones and A. Melissinos (WSPC, Singapore, 1990).

60. Dark Matter Candidates

Michael S. Turner

in Proceedings of the Srd CERN/ESO Symposium on particle Physics and Cosmology, ed. M. Caffo et al. (Kluwer, Dordrecht, 1989).

61. Lectures on Particle Physics and Cosmology

Michael S. Turner

in Proceedings of the Brown University TASI (WSPC, Singapore, 1990).

62. Lectures on Particle Physics and Cosmology

Michael S. Turner

in Proceedings of the 1st Puerto Rico Winter School on Cosmology and Particle Physics (1989).

63. Lectures on Cosmology and Particle Physics

Michael S. Turner

in Proceedings of the South African Summer School in Theoretical Physics (1989).

64. Lectures on Cosmology and Particle Physics

Michael S. Turner

in Proceedings of the 2nd CCAST Summer School (1989).

65. Toward Inflationary Paradigm: Lectures on Inflationary Cosmology

Michael S. Turner

Nuovo Cimento Redazione

66. Searching for $C P$ Violation in 'Charge-Blind' Jets

Mark Kamionkowski

Physical Review D 41, 1672 (1990).

67. Unitary Limits on the Mass and Radius of Dark Matter Particles 
Kim Griest and Mark Kamionkowski

Physical Review Letters 64, 615 (1990).

68. Supersymmetric Dark Matter Above the $W$ Mass

Kim Griest, Mark Kamionkowski, and Michael S. Turner

Physical Review D 41, 3565 (1990).

69. Astrophysical and Cosmological Constraints on Neutrino Properties

E.W. Kolb, D.N. Schramm, and M.S. Turner

in Neutrino Physics, ed. K. Winter (Cambridge Univ. Press, Cambridge, 1991).

70. Cosmology and the Neutron Lifetime

L. Kawano and D.N. Schramm

Nucl. Inst. Meth. A 284, 84 (1989).

71. False Vacuum Decay in Jordan-Brans-Dicke Cosmologies

E.V: Kolb, R. Holman, S.L. Vadas, Y. Wang, and E.J. Weinberg

Physics Letters B 237, 37 (1990).

72. Thermal Relics: Do We Know Their Abundances?

M. Kamionkowski and Michael S. Turner

Physical Review $D$, in press (1990).

73. Axions and SN1987A: The Trapped Regime

A. Burrows, M.T. Ressell, and Michael S. Turner

Physical Review $D$, in press (1990).

74. Time Delays in Gravitational Lenses from Large-scale Structure and Gravitational Waves

J.A. Frieman, N. Kaiser, and Michael S. Turner

Physical Review D, submitted (1990).

75. Periodic Signatures for the Detection of Cosmic Axions

Michael S. Turner

Physical Review D, in press (1990).

76. Cosmological Baryon and Lepton Number in the Presence of Electroweak Fermion Number Violation

J.A. Harvey and Michael S. Turner

Physical Review D, in press (1990).

77. Origin of Density Fluctuations in Extended Inflation

E.W. Kolb, D.S. Salopek, and Michael S. Turner

Physical Review D, in press (199C).

78. Microwave Distortions from Collapsing Domain-wall Bubbles

Michael S. Turner, R. Watkins, and L.M. Widrow

Astrophysical Journal (Letters), submitied (1990).

79. Inflationary Axion Cosmology

Michael S. Turner and Frank Wilczek

Physical Revieu Letters, submitted (1990). 
80. Relic Gravitational Waves and Extended Inflation Michael S. Turner and Frank Wilczek Physical Review Letters, submitted (1990).

81. Can Oscillating Physics Explain an Apparently Periodic Universe? Christopher T. Hill, Paul J. Steinhardt, and Michael S. Turner Physics Letters B, in press (1990).

82. Coherent Peculiar Velocity Fields and Periodic Red Shifts Christopher T. Hill, Paul J. Steinhardt, and Michafil S. Turner Astrophysical Journal (Letters), submitted (1990).

83. Oscillating Scalar Fields and Hubble Constants Michael S. Turner and C.T. Hill FERMILAB-PUB-90/103-T (1990).

84. A Distinctive Positron Feature from Heavy Neutralino Annihilation Mark Karnionkowski and Michael S. Turner Physical Review D, submitted (1990).

85. Results of a Telescope Search for eV-mass Axions M. Bershady, M.T. Ressell, and Michael S. Turner Physical Review Letters, submitted (1990).

86. The Early Universe Michael S. Turner Proceedings of GR 12, ed. N. Ashby (1990).

87. Dark Matter in the Universe Michael S. Turner Proceedings of the Nobel Symposium on the Birth and Early Evolution of the Universe, ed. B.-S. Skagerstam (1990).

88. Semiclassical Stability in Multidimensional Cosmologies

L. Amendola, E.W. Kolb, M. Litterio, and F. Occhionero

Physical Review D, in press (1990).

89. Gravitational Couplings of the Inflaton in Extended Inflation R. Holman, E.W. Kolb, and Y. Wang Physical Review Letters 65, 17 (1990).

90. Topological Defects in Extended Inflation E. J. Copeland, E.W. Kolb, and A. R. Liddle Physical Review D, in press (1990).

91. Baryogenesis in Extended Inflation I. Baryogenesis via Production and Decay of Supermassive Bosons

J. D. Barrow, E. J. Copeland, E.W. Kolb, and A. R. Liddle Physical Review D, submitted (1990).

92. Baryogenesis in Extended Inflation II. Baryogenesis via Production and Evaporation of Primordial Black Holes 
J. D. Barrow, E. J. Copeland, E.W. Kolb, and A. R. Liddle

Physical Review D, submitted (1990).

93. Kaluza-Klein Extended Inflation

R. Holman, E.W. Kolb, S. L. Vadas, and Y.Wang

Physical Review D, submitted (1990).

94. False-Vacu ım Decay in Generalized Extended Inflation

R. Holman, E.W. Kolb, S. L. Vadas, and Y. Wang

Physics Letters $B$, in press (1990).

95. The Imperfect Universe

E.W. Kolb

in Proceedings of XXth Yamada Conference, eds. S. Hayakawa and K. Sato (WSPC, Singapore)

96. CP Violation in the Early Univerı

E.W. Kolb

in CP Violation, ed. Tran Thanh Van (Editions Frontieres, 1989).

97. Neutrino Cosmology and Astrophysics

E.W. Kolb

in Proceedings of the Second International Meeting on Neutrino Telescopes, ed. M.

Bldo-Ceolin

98. Cosmic Phase Separation with Subcritical Bubbles

M. Gleiser, E.W. Kolb, and R. Watkins

Physical Review D, submitted (1990).

99. Isocurvature Fluctuations from Goldstone Bosons

J.A. Frieman, M.S. Turner, and R. Watkins

Physical Review D, submitted (1990).

100. Evolution of Light Domain Walls Interacting with Dark Matter

A. Massarotti

Physical Review D, in press (1990).

101. Formation of Domain Walls in the Early Universe

L. Kaviano

Physical Review D 41, 1013 (1990). 

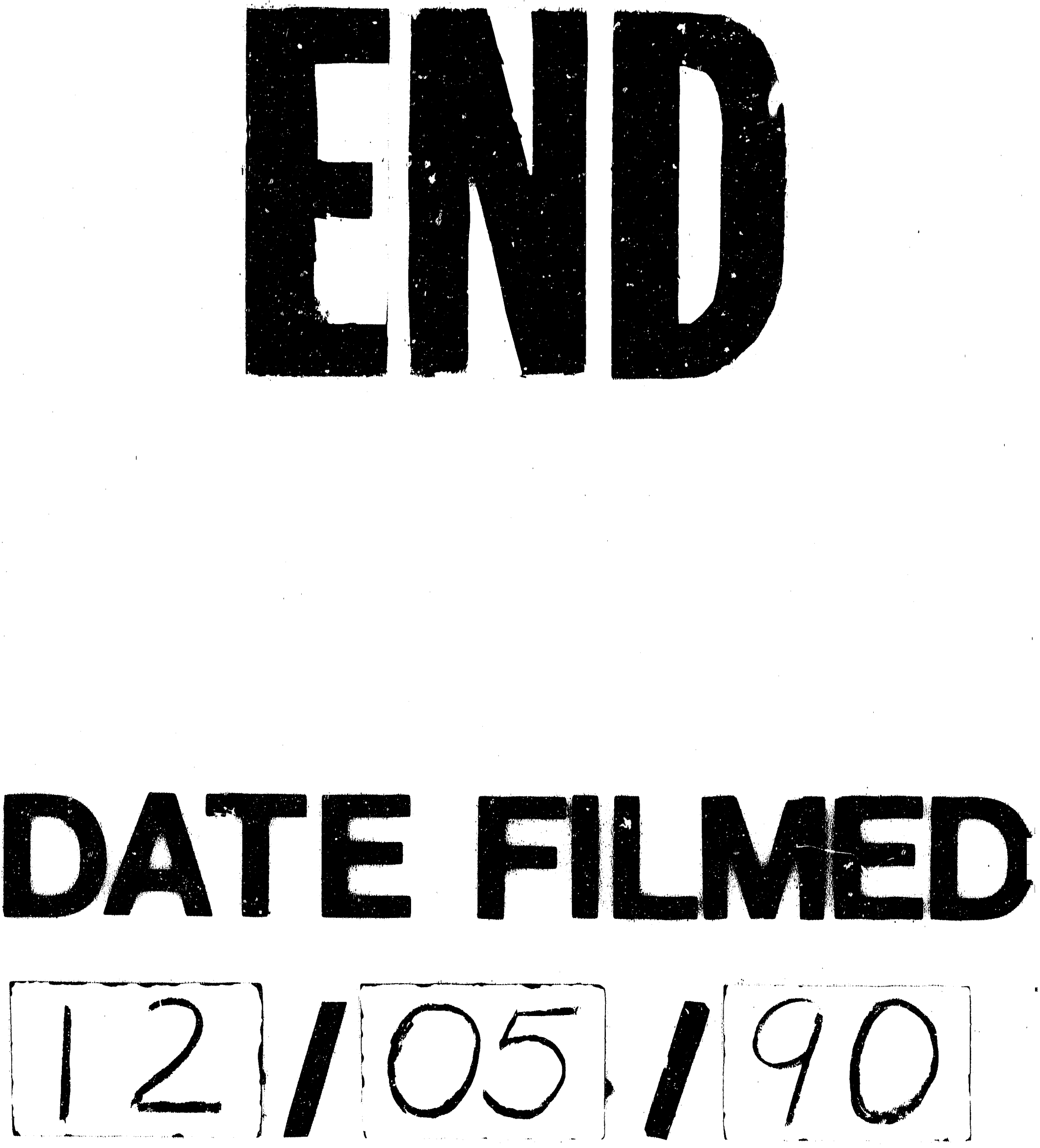
